University of Nebraska - Lincoln

DigitalCommons@University of Nebraska - Lincoln

Faculty Publications, Department of Physics and Astronomy

Research Papers in Physics and Astronomy

4-2004

\title{
Low-Pressure Source of Slow Metastable Rare Gas Atoms
}

\author{
M. H.L. van der Velden \\ Eindhoven University of Technology \\ Herman Batelaan \\ University of Nebraska - Lincoln, hbatelaan@unl.edu \\ E. te Sligte \\ Eindhoven University of Technology \\ H. C.W. Beijerinck \\ Eindhoven University of Technology \\ E. J.D. Vredenbregt \\ Eindhoven University of Technology, E.J.D.Vredenbregt@tue.nl
}

Follow this and additional works at: https://digitalcommons.unl.edu/physicsfacpub

Part of the Physics Commons

van der Velden, M. H.L.; Batelaan, Herman; te Sligte, E.; Beijerinck, H. C.W.; and Vredenbregt, E. J.D., "LowPressure Source of Slow Metastable Rare Gas Atoms" (2004). Faculty Publications, Department of Physics and Astronomy. 118.

https://digitalcommons.unl.edu/physicsfacpub/118

This Article is brought to you for free and open access by the Research Papers in Physics and Astronomy at DigitalCommons@University of Nebraska - Lincoln. It has been accepted for inclusion in Faculty Publications, Department of Physics and Astronomy by an authorized administrator of DigitalCommons@University of Nebraska Lincoln. 


\title{
Low-pressure source of slow metastable rare gas atoms
}

\author{
M. H. L. van der Velden, H. Batelaan, ${ }^{\text {a) }}$ E. te Sligte, H. C. W. Beijerinck, \\ and E. J. D. Vredenbregt ${ }^{\text {b) }}$ \\ Physics Department, Eindhoven University of Technology, P.O. Box 513, 5600 MB Eindhoven, Netherlands
}

(Received 7 October 2003; accepted 11 January 2004; published 16 March 2004)

\begin{abstract}
We investigate the properties of a commercial inverted magnetron pressure gauge for use as a source of slow metastable rare gas atoms. We find that the velocity distribution of the atoms as well as the pressure dependence of the output flux agree with a simple model. This shows that the low-velocity output of the source is enhanced over the Maxwell-Boltzmann form due to a velocity-dependent excitation probability. For argon, the center-line intensity per unit area of the source is measured to be greater than $4.2 \times 10^{15} \mathrm{Ar} 1 s_{5}$ atoms $/\left(\mathrm{s} \mathrm{sr} \mathrm{m}^{2}\right)$ at a pressure of $23 \mathrm{mPa}$. When observing the entire source area, the center-line intensity is at least $2.6 \times 10^{11} \mathrm{Ar} 1 s_{5}$ atoms/(s sr). (C) 2004 American Institute of Physics. [DOI: 10.1063/1.1666988]
\end{abstract}

\section{INTRODUCTION}

Metastable rare gas ( $\mathrm{RG})$ atoms find applications in many types of experiments, such as ultracold plasma research, ${ }^{1}$ Bose-Einstein condensation ${ }^{2}$ and collision studies. ${ }^{3}$ They are normally produced in gas discharges. The usual low-energy metastable RG atom source consists of a supersonic expansion through a nozzle, through which a discharge current is drawn. ${ }^{4}$ This type of source is characterized by a narrow velocity distribution centered at a high average speed $u_{s}$ that is related to the source temperature $T$ by $u_{s}$ $\lesssim \sqrt{5 k_{\mathrm{B}} T / M}$, where $k_{\mathrm{B}}$ is Boltzmann's constant and $M$ the atomic mass. ${ }^{5}$ The large average velocity is undesirable for laser slowing and trapping experiments ${ }^{6}$ since it leads to a large stopping length (which is proportional to $u_{s}^{2}$ ) and large beam divergence of the slowed beam (proportional to $\sqrt{u}_{s}$ ). Thus, there has been a certain interest in reducing the average velocity, for example, by cooling the source with liquid nitrogen ${ }^{7}$ or even liquid helium. ${ }^{8}$ These methods are somewhat cumbersome, however, and in addition do not work for the heavier RGs since these would simply condense at such low temperatures.

A different way of providing low-velocity atoms is to eliminate the supersonic character of the expansion and, instead, create a thermal velocity distribution. Such a thermal source not only leads to a lower average velocity $u_{\text {th }}$ $=\sqrt{4 k_{\mathrm{B}} T / M \pi}<u_{s}$, but also to a larger spread, so that atoms with velocities far below the average become available. With alkali-metal atoms, capturing atoms from the low-velocity tail of a $300 \mathrm{~K}$ thermal distribution is a popular way to load a so-called vapor cell magneto-optical trap ${ }^{9}$ or to create a low-velocity atomic beam. ${ }^{10}$

To extract slow atoms from such a source, it has to operate at low pressure, because otherwise the slow atoms are lost from the extracted atomic beam by collisions with the surrounding gas. For metastable RG sources, this creates a

\footnotetext{
${ }^{a)}$ Current address: Department of Physics, University of Nebraska—Lincoln, Lincoln, NE 68558-0111.

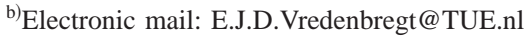

quandary: a discharge is required, but in the low-pressure regime, the mean free path for both neutral and charged particles is too large to sustain one. However, in a magnetic field, electrons may be trapped for a long time, such that sufficient electron density builds up to allow a discharge to run. A commercial device that operates precisely in this regime is the traditional inverted magnetron ionization gauge. $^{11}$

In its simplest form, an inverted magnetron gauge consists of a central anode pin, surrounded by a cylindrical cathode with (usually perforated) end-plates (see Fig. 1). The cathode-to-anode voltage is typically several kilovolts. A large magnetic field of order $1 \mathrm{kG}$ is directed along the symmetry axis of the device, and is therefore perpendicular to the local electric field everywhere. In such a crossed-fields configuration, electrons spiral around the magnetic field lines on their voyage to the anode. This leads to a substantial increase of their residence time, with a corresponding increase in the probability of their colliding with atoms and molecules traversing the gauge, causing ionization. In this way, a discharge can be maintained at pressures as low as $10^{-11} \mathrm{~Pa}^{11}$ The pressure is then inferred from a measurement of the discharge current.

In this article, we investigate the performance of such a gauge as a source of slow metastable RG atoms. We report the velocity distribution and total flux of metastable atoms that can be extracted from this device. We also study the effect of collisions with ground-state atoms by its influence on the dependence of these quantities on the ambient source pressure with the aim of finding the optimum conditions for extracting slow atoms. These studies are part of an effort to create a table-top trap for metastable RG atoms. ${ }^{12}$

\section{EXPERIMENTAL PROCEDURE}

Figure 2 shows the experimental setup used in our study. The source is an Edwards AIM-S-NW25 pressure gauge, and is mounted at the far end of a 170-mm-long vacuum chamber. The source is mounted $4 \mathrm{~mm}$ off-center from the axis of the setup to avoid looking at the central electrode. We have 


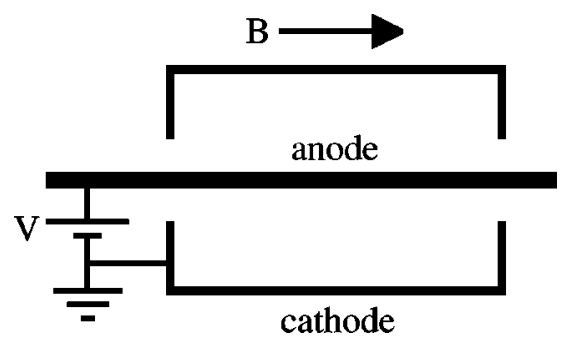

FIG. 1. Schematic view of inverted magnetron gauge, consisting of central anode pin, cylindrical cold cathode, and axial magnetic field.

modified the source by replacing the exit plate by a $60 \%$ open, stainless steel wire mesh. The gauge is used both as source and as pressure gauge. Argon gas is fed through a needle valve into the source chamber, which is not actively pumped. Atoms exit the chamber through a 2-mm-diam hole in the wall opposite from the source and enter a $430-\mathrm{mm}$ long differential pumping stage, pumped by a $500 \ell / \mathrm{s}$ turbomolecular pump. Next, they pass though a 6-mm-diam, 60$\mathrm{mm}$-long flow resistance whose exit is located $600 \mathrm{~mm}$ from the source. Here, they enter the detection chamber, which is pumped by a $15 \ell / \mathrm{s}$ ion-getter pump.

The detector consists of a glass plate covered with a thin indium tin oxide (ITO) layer, located $1040 \mathrm{~mm}$ from the exit of the source. Metastable atoms can free electrons from the ITO plate through an Auger process. The resulting current is measured with a Keithley $610 \mathrm{C}$ electrometer, which provides an absolute measure for the total flux of metastable atoms. The freed electrons are accelerated toward a Burle 4039-EIC channeltron electron multiplier operated in pulse counting mode.

Besides metastable argon atoms, the source also produces UV photons. To discern the two, the atomic beam is crossed by a $\approx 3$-mm-wide laser beam from a home-built diode-laser system with grating feedback. ${ }^{13}$ The laser is stabilized to the $1 s_{5} \rightarrow 2 p_{8}$ transition in argon at $801.479 \mathrm{~nm}$ using the absorption in a discharge cell. The laser has sufficient intensity to completely deplete the metastable $1 s_{5}$ state by transferring the population to the ground state through the intermediate $2 p_{8}$, so that it can be used to modulate the presence of metastable atoms. We have no means of modu-

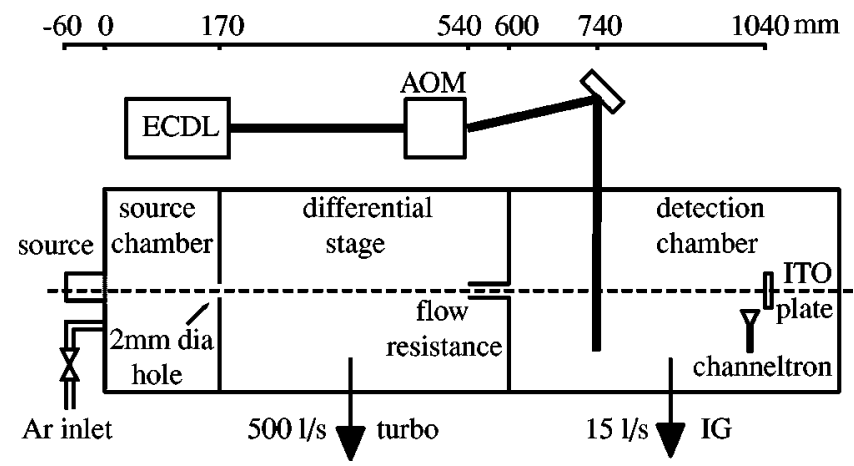

FIG. 2. Schematic view of experimental apparatus, comprised of ionization gauge in source chamber, differential pumping stage, and detection chamber with ITO plate and channeltron. The population of metastable atoms is modulated with an extended-cavity diode laser (ECDL), which is also used for time-of-flight measurements in combination with an acousto-optical modulator (AOM). Relevant lengths are indicated. lating the population of the other metastable state, $\operatorname{Ar} 1 s_{3}$, so we cannot measure its contribution to the signal. The ratio between the $1 s_{3}$ and $1 s_{5}$ states is typically 0.2 for other discharge sources. The laser beam is run through an acoustooptical modulator, which acts as a high-speed shutter. The crossing between laser and atomic beam occurs at $(300 \pm 5)$ $\mathrm{mm}$ before the ITO plate.

To obtain the velocity distribution of the metastable atoms, we take time-of-flight measurements ${ }^{14}$ in which we use the laser beam as a chopper and measure the count rate on the channeltron as the response. Because of the high background signal due to UV photons, we use the pseudorandom modulation method ${ }^{14}$ to obtain a sufficient signal-to-noise ratio in a reasonable time. We use a $200 \mu$ s time base for the modulation, giving a velocity resolution of typically $10 \%$.

\section{MODEL OF PERFORMANCE}

At the normal operating pressures of an inverted magnetron gauge (below $10 \mathrm{mPa}$ ), we are in the molecular flow regime. As a consequence, the gas is in thermal equilibrium with the surrounding walls and its velocity distribution $P(\mathbf{v})$ will be Maxwell-Boltzmann:

$$
P(\mathbf{v}) d^{3} \mathbf{v}=\frac{4}{\sqrt{\pi}}(v / \alpha)^{2} e^{-(v / \alpha)^{2}} \frac{d v}{\alpha} \frac{d \Omega}{4 \pi},
$$

with $v$ the absolute value of the velocity vector $\mathbf{v}$ and $\Omega$ representing the angular coordinates. Here, $\alpha=\sqrt{2 k_{\mathrm{B}} T / M}$ is the most probable or thermal velocity, which is equal to 353 $\mathrm{m} / \mathrm{s}$ for argon at $300 \mathrm{~K}$. Ground-state atoms that traverse the magnetron gauge have only a small probability of colliding with an electron and ending up in a metastable state. In this case, the velocity-dependent excitation probability $\eta(v) \ll 1$ will be proportional to the time spent in the gauge, and therefore inversely proportional to the atom's velocity, so that we write

$$
\eta(v)=\eta_{\alpha} \alpha / v .
$$

Due to the geometry of the gauge, $\eta$ in fact will also depend on the direction of motion though the gauge, but since we will be interested mainly in the flux of atoms leaving the source in a narrow cone centered around the forward direction, we will ignore this fact. Here, we do not attempt to model the dependence of $\eta$ on discharge voltage or magnetic field, but address this issue experimentally (see Fig. 6).

Since the gauge is mounted in a separate vacuum chamber to allow for differential pumping, metastable atoms created in the source will have to travel a distance $L$ $=215 \mathrm{~mm}$ (including an average distance of $45 \mathrm{~mm}$ traveled inside the source itself) through the gas in the source chamber before they can exit through an orifice in the wall opposite from where the gauge is mounted. On this path, they may collide with ground-state atoms and effectively be lost. Using Beer's law, we can describe this with a transmission coefficient $\Theta(v)$ :

$$
\Theta(v)=\exp [-n L\langle Q(g) g\rangle / v],
$$

where $n$ is the density of ground-state atoms, $g$ the relative velocity of the collision, $Q(g)$ the elastic cross section, and 


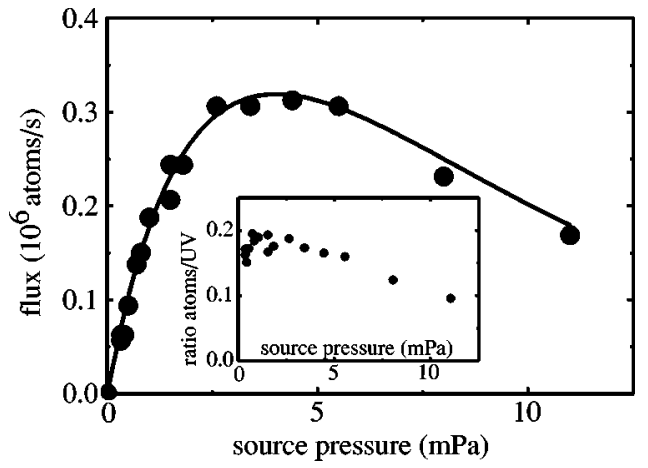

FIG. 3. Closed circles: measured total flux of $\operatorname{Ar} 1 s_{5}$ atoms versus source pressure, assuming unit detection efficiency. Solid line: fitted curve corresponding to Eq. (7). The inset shows the ratio of the current due to metastable atoms to that of UV photons.

\langle\rangle stands for averaging over the velocity distribution of the scatterers. The cross section $Q$ may be derived from the Van der Waals coefficient $C_{6}$ for the interaction of the groundstate and metastable atoms, as ${ }^{15}$

$$
Q=8.08\left(C_{6} / \hbar g\right)^{2 / 5}=Q_{\alpha}(\alpha / g)^{2 / 5} .
$$

For argon-metastable-argon collisions, $\quad C_{6}=28$ $\times 10^{-78} \mathrm{~J} \mathrm{~m}^{6}$ (see Ref. 15) giving $Q_{\alpha}=730 \times 10^{-20} \mathrm{~m}^{2}$. The transmission coefficient can alternatively be written as

$$
\Theta(v)=\exp \left[-f(v) \frac{\alpha}{v} \frac{p}{p_{0}}\right],
$$

in which $p_{0}=k_{\mathrm{B}} T / L Q_{\alpha}=2.6 \mathrm{mPa}$, and some elementary algebra gives (within 2.5\% accuracy) $f(v) \approx\left[f_{0}^{10 / 3}\right.$ $\left.+(v / \alpha)^{2}\right]^{3 / 10}$ with $f_{0}=8 \Gamma(4 / 5) / 5 \sqrt{\pi}=1.051$.

The particle detector installed in our apparatus measures flux. To arrive at an equation for the velocity distribution of the flux $\psi(v)$ of metastable atoms measured downstream on the detector, we note that in the experiment, only a small solid angle $\Omega=68 \times 10^{-6}$ sr of the angular distribution contributes to the signal (limited by the flow resistance), and only a limited area $A=5.0 \mathrm{~mm}^{2}$ of the source can be observed from the detector (see Fig. 2). In that case, $\psi$ is equal to $\eta(v) n v A \Omega \Theta(v) P(v)$, so that we obtain

$$
\psi(v)=\frac{4}{\sqrt{\pi}} \eta_{\alpha} \frac{p}{k_{\mathrm{B}} T} A \frac{\Omega}{4 \pi} \Theta(v)(v / \alpha)^{2} e^{-(v / \alpha)^{2}} .
$$

The total flux $\Psi$ of atoms arriving at the detector is then simply

$$
\Psi=\int_{0}^{\infty} \psi(v) d v
$$

This has a broad maximum as a function of pressure occurring at $p \approx 0.725 p_{0}$, where $\Psi \approx 0.0210 \eta_{\alpha} \alpha A \Omega p_{0} / k_{\mathrm{B}} T$. Finally, we define the center-line intensity as $\Psi / A \Omega$ to facilitate a direct comparison with other sources.

\section{MEASURED PERFORMANCE}

In Fig. 3, we show the metastable atom flux deduced from the current measured on the ITO plate as a function of source pressure. This flux is obtained from the difference

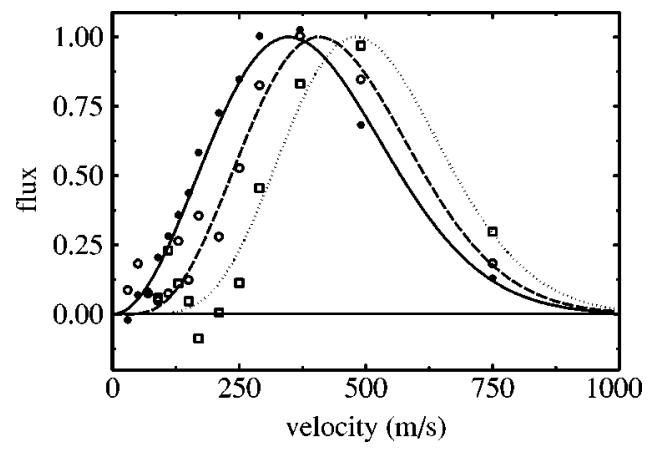

FIG. 4. Measured velocity dependence of the metastable atom flux for three different source pressures: $0.1 \mathrm{mPa}$ (solid line, filled circles), $4 \mathrm{mPa}$ (dashed line, open circles), and $11 \mathrm{mPa}$ (dotted line, open squares). The lines are curve fits to Eq. (6).

between the currents measured with chopper laser on and off. To translate current to atom flux, we assume unit detection efficiency for $\operatorname{Ar} 1 s_{5}$, so that we obtain a lower limit. Given the detection efficiency reported by Hotop, ${ }^{16}$ the actual flux is probably a factor of 5 higher than shown in Fig. 3. We observe a strong increase of the signal with pressure up to $\approx 5 \mathrm{mPa}$, where the atom flux saturates at a value of 0.3 $\times 10^{6} \mathrm{Ar} 1 s_{5}$ atoms/s. At this point, the center-line intensity of the source is $0.89 \times 10^{15}$ atoms $/\left(\mathrm{s} \mathrm{sr} \mathrm{m}^{2}\right)$. In Fig. 3, we also show that the measured curve compares very well with Eq. (7) if we assume a collision cross section $Q_{\alpha}=280$ $\times 10^{-20} \mathrm{~m}^{2}$ (reference pressure $p_{0}=6.9 \mathrm{mPa}$ ) and a pressure-independent excitation probability $\eta_{\alpha}=7.2 \times 10^{-5}$. The fact that $Q_{\alpha}$ comes out substantially smaller than the value measured by Kerstel et al. ${ }^{15}$ is easily explained by considering that small-angle elastic collisions do not have to lead to a loss of atoms from the beam and should therefore be excluded from the cross section. We also note that, indeed, $\eta_{\alpha} \ll 1$, validating the approach in Sec. III. Figure 3 also shows the ratio of the atom signal compared to that for UV photons. At low pressure, this reaches about 20\%, while at higher pressure it drops off because of the loss of atoms by collisions.

In Fig. 4, we show the distribution of the flux over velocity as measured with the time-of-flight technique, for three different source pressures. At the lowest pressure (0.1 $\mathrm{mPa}$ ), the measured distribution fits almost perfectly with Eq. (6) for the expected thermal velocity of $\alpha=353 \mathrm{~m} / \mathrm{s}$ and unit transmission $\Theta(v)=1$. At higher pressures, the data also fit Eq. (6), but now the loss of atoms due to collisions is clearly visible by the absence of low-velocity atoms, effectively shifting the maximum of the flux distribution to higher velocities. From the fits, we deduce the product $n L Q_{\alpha}$ $=p / p_{0}$, and plot this in Fig. 5. Consistent with the model, we find a linear dependence on source pressure. The corresponding cross section $Q_{\alpha}=(292 \pm 20) 10^{-20} \mathrm{~m}^{2} \quad\left(p_{0}=6.6\right.$ $\pm 0.4 \mathrm{mPa}$ ) is in agreement with the value deduced from the total flux measurement. These results indicate that $\eta_{\alpha}$ is independent of pressure, probably because the electron density is independent of pressure in this discharge regime. ${ }^{17}$

We have also looked into the dependence of the flux on the discharge voltage $V$ and axial magnetic field $B$ of the gauge by replacing the standard electronics and magnet 


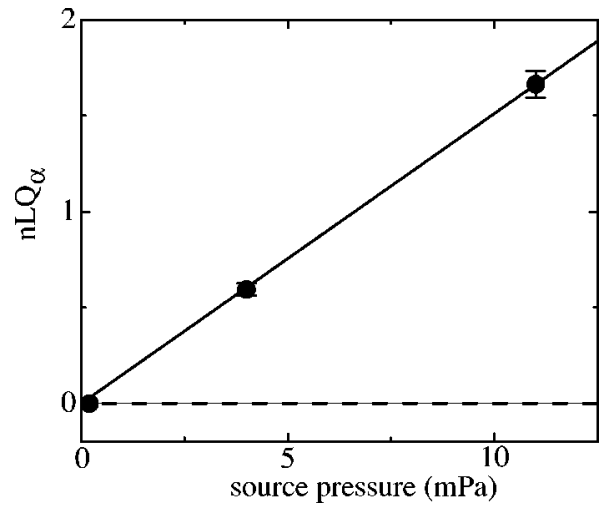

FIG. 5. Values of $n L Q_{\alpha}$ resulting from fitting curves to the data in Fig. 4 versus source pressure. From the observed linear behavior, the cross section for collisional loss is derived.

housing by an adjustable high-voltage supply and magnet coil. The magnetic-field dependence plotted in Fig. 6 shows an optimum at $850 \mathrm{G}$; this is reminiscent of the change-over from a low-field to a high-field discharge mode as investigated by Schuurman. ${ }^{17}$ The optimum then corresponds to the maximum in the discharge current that occurs at the transition point. The optimum field is precisely the field present in the commercial device. As also shown in Fig. 6, the flux increases linearly with voltage in the range we considered, but seems to drop off at voltages higher than the $3 \mathrm{kV}$ of the commercial device. Given the magnetic-field dependence, this is to be expected since the low- to high-field transition is also crossed by reducing the voltage at fixed magnetic field. We conclude that the commercial device is already welloptimized for our purpose. A simultaneous increase of both $V$ and $B \propto \sqrt{V}$ may, however, further increase the yield. ${ }^{17}$

\section{DISCUSSION}

Since the measured velocity dependence of the flux agrees with Eq. (6), we conclude that the probability of exciting the metastable state is indeed inversely proportional to velocity. This means that slow atoms are relatively more abundant than in a purely thermal source, such as that commonly used to produce alkali-metal beams. As such, this enhances the usefulness of the ionization gauge as a source of slow atoms.

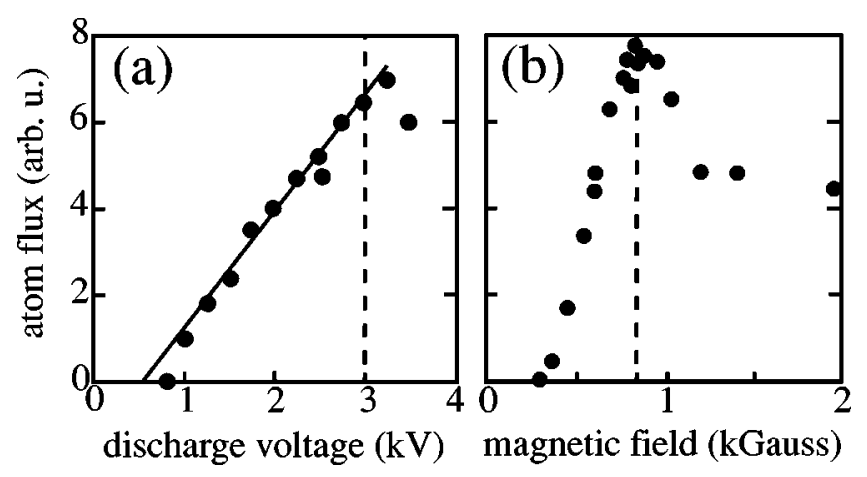

FIG. 6. Dependence of the flux of metastable atoms (a) on discharge voltage and (b) applied magnetic field. Both data sets were taken at a pressure of $0.15 \mathrm{mPa}$. The dashed line indicates the operating parameters of the commercial device; the solid line in (a) guides the eye.
Given the results, we can also calculate the total flux for the case in which the source would be mounted directly in front of the 2-mm-diam hole in the source vessel, thereby eliminating the loss of slow atoms by collisions in the source chamber. In this case, we can replace the length $L$ by the average distance an atom has to traverse inside the source itself after it has been excited, which gives $L=45 \mathrm{~mm}$ for the Edwards AIM gauge. This should shift the pressure where the maximum occurs and, consequently, the achieved centerline intensity, by a factor of 4.8 . We conclude that in this case, this type of source is capable of producing 4.2 $\times 10^{15} \mathrm{Ar} 1 s_{5}$ atoms $/\left(\mathrm{s} \mathrm{sr} \mathrm{m}^{2}\right)$ at this pressure. We note once more that this number is probably substantially higher since we have assumed unit detection efficiency for our measurements. Similar results should be expected for other rare gases. We speculate that comparable results may be obtained with other atomic and molecular species, such as $\mathrm{Ca}$ and $\mathrm{N}_{2}$.

In the experiments reported here, we have observed only a small area (diameter $2.6 \mathrm{~mm}$ ) of the output surface of the source. We have not investigated how the output might depend on the position of the observation area within the diameter of the source opening. Since, however, our source is mounted $4 \mathrm{~mm}$ off-center, one may expect the measured flux to hold at least over a 2.6-mm-wide, 8 -mm-diam ring, that is, for $A=32 \mathrm{~mm}^{2}$. In this case, the total output that can be collected from the source would be 2.6 $\times 10^{11} \mathrm{Ar} 1 s_{5}$ atoms/( $\mathrm{s} \mathrm{sr}$ ).

Using the measured values of $\eta_{\alpha}$ and $p_{0}$ for $L$ $=0.215 \mathrm{~m}$, we can predict the useful flux of atoms that we can expect to collect in our table-top trap for metastable argon atoms. ${ }^{12}$ Simulations have shown ${ }^{12,18}$ that we can collect all atoms emanating from the source within a solid angle of $\approx 0.07 \mathrm{sr}$ and with velocities lower than $200 \mathrm{~m} / \mathrm{s}$. Now, using Eq. (7), but with the upper limit replaced by $200 \mathrm{~m} / \mathrm{s}$, we find an optimum value of $\approx 2.5 \times 10^{7}$ atoms $/ \mathrm{s}$ in this velocity range that can be loaded into our magneto-optical atom trap. The optimum requires a source pressure of $1.7 \mathrm{mPa}$. For the setup in Fig. 2, this allows pressures below $100 \mathrm{nPa}$ in the trapping chamber. Given the typical lifetime of such traps under these conditions, we can expect to operate traps with a population of around $10^{8}$ atoms; that is, in the range where vapor-cell traps for alkali-metal atoms normally operate.

Finally, we remark that an advantage of using this source is that it is a relatively in expensive, commercially available product that does not require any modifications in a machine shop.

\section{ACKNOWLEDGMENTS}

The authors thank D. Driessen and R. van Ratingen for contributions to this work, as well as L. van Moll, J. van de Ven, and M. de Koning for technical assistance. This work was partially funded by the Netherlands Foundation for Fundamental Research on Matter (FOM).

${ }^{1}$ T. C. Killian, V. S. Ashoka, P. Gupta, S. Laha, S. B. Nagel, C. E. Simien, S. Kulin, S. L. Rolston, and S. D. Bergeson, J. Phys. A 36, 6077 (2003).

${ }^{2}$ A. Robert, O. Sirjean, A. Browaeys, J. Poupard, S. Nowak, D. Boiron, C. I. Westbrook, and A. Aspect, Science 292, 461 (2001). 
${ }^{3}$ J. Leonard, M. Walhout, A. P. Mosk, T. Mueller, M. Leduc, C. CohenTannoudji, Phys. Rev. Lett. 91, 073203 (2003).

${ }^{4}$ For a review, see T. J. Gay, in Atoms and Molecules, Atomic, Molecular and Optical Physics Vol. 29b (Academic, New York, 1996). A recent list of references may be found in W. Lu, M. D. Hoogerland, D. Milic, K. G. H. Baldwin, and S. J. Buckman, Rev. Sci. Instrum. 72, 2558 (2001), and in C. Y. Chen, K. Bailey, Y. M. Li, T. P. OConnor, Z.-T. Lu, X. Du, L. Young, and G. Winkler, ibid. 72, 271 (2001).

${ }^{5}$ D. R. Miller, in Atomic and Molecular Beam Methods edited by G. C. Scoles (Oxford University Press, Oxford, 1988), Vol. 1.

${ }^{6} \mathrm{H}$. Metcalf and P. van der Straten, Laser Cooling and Trapping (Springer, New York, 1999).

${ }^{7}$ J. Kawanaka, M. Hagiuda, K. Shimizu, F. Shimizu, and H. Takuma, Appl. Phys. B: Photophys. Laser Chem. 56, 21 (1993).

${ }^{8}$ G. R. Woestenenk, J. W. Thomsen, M. van Rijnbach, and P. van der Straten, Rev. Sci. Instrum. 72, 3842 (2001).

${ }^{9}$ M. Stevens and C. Wieman, Phys. Rev. Lett. 72, 3787 (1994).
${ }^{10}$ J. Schoser, A. Batär, R. Löw, V. Schweikhard, A. Grabowski, Yu. B. Ovchinnikov, and T. Pfau, Phys. Rev. A 66, 023410 (2002).

${ }^{11}$ For a review, see P. A. Redhead, J. Vac. Sci. Technol. A 12, 904 (1994), or A. Berman, Total Pressure Measurements in Vacuum Technology (Academic, New York, 1985).

${ }^{12}$ E. J. D. Vredenbregt, K. A. H. van Leeuwen, and H. C. W. Beijerinck, Opt. Commun. 147, 375 (1998).

${ }^{13}$ C. E. Wieman and L. Hollberg, Rev. Sci. Instrum. 62, 1 (1991).

${ }^{14}$ D. J. Auerbach, in Atomic and Molecular Beam Methods edited by G. C. Scoles (Oxford University Press, Oxford, 1988), Vol. 1.

${ }^{15}$ E. R. T. Kerstel, C. P. J. W. van Kruysdijk, J. C. Vlugter, and H. C. W. Beijerinck, Chem. Phys. 121, 211 (1988).

${ }^{16} \mathrm{H}$. Hotop, in Atoms and Molecules, Atomic, Molecular and Optical Physics, Vol. 29b (Academic, New York, 1996), p. 191.

${ }^{17}$ W. Schuurman, Physica (Amsterdam) 36, 2003 (1967)

${ }^{18}$ M. H. L. van der Velden, M.Sc. thesis, Eindhoven University of Technology, Internal Report AQT/03-07. 